\title{
Dynamic Property Rights Spectrum Access: Flexible Ownership Based Spectrum Management
}

\author{
Omer Ileri \\ WINLAB, \\ Rutgers University \\ 671 Route $1 \mathrm{~S}$ \\ North Brunswick, NJ 08902 \\ Email: omeriler@winlab.rutgers.edu
}

\author{
Dragan Samardzija \\ Bell Laboratories, Lucent Technologies \\ 791 Holmdel-Keyport Road \\ Holmdel, NJ 07733 \\ Email: dragan@alcatel-lucent.com
}

\author{
Narayan B. Mandayam \\ WINLAB, \\ Rutgers University \\ 671 Route $1 \mathrm{~S}$ \\ North Brunswick, NJ 08902 \\ Email: narayan@winlab.rutgers.edu
}

\begin{abstract}
In this paper we develop a framework for operator competition based on short term ownership of spectrum resources and devise a dynamic spectrum access method called "Dynamic Property Rights Spectrum Access (D-Pass)”. In the D-Pass model, spectrum portions are allocated to operators on a short term basis (few sessions) by a SPS (Spectrum Policy Server) that serves as a controller/enforcer as well as a clearinghouse for spectrum allocations. Prior to each short term allocation, the SPS optimally determines a specific partition of spectrum resources among the operators to maximize a system related objective function. The operators are charged by the SPS for the amount of spectrum they are allocated. Given the spectrum allocation, the operators compete with each other for users present in the system through demand responsive pricing, in the form of an iterative bidding scheme reminiscent of simultaneous ascending auctions. At every iteration, the operators make rate and price offers for each user considering the bandwidth allocated to them and their costs associated with serving the users. The users respond by declaring the probabilities with which they will accept the service offers made. We consider two different objective functions for the SPS to maximize while determining the exact partition of the spectrum resources: (1) the total expected bandwidth utilization, (2) the minimum acceptance probability that a user accepts the offered service. We demonstrate several tradeoffs between these objectives through numerical experiments and illustrate the effect of bandwidth cost on these tradeoffs. Our results also suggest that employing short term allocation of resources could potentially lead to performance gains as opposed to static allocation of resources, especially in regimes where the bandwidth is relatively expensive.
\end{abstract}

\section{INTRODUCTION}

The current spectrum governance scheme employed by the FCC is in the form of a command and control approach in which access to large portions of spectrum is under strict governmental control. The spectrum is allocated for predefined services with strict rules for physical layer technologies employed. The spectrum allocation decisions have mostly been made with the aim of reducing unwanted interference among communicating devices and based on the radio communication technologies employed at the time of the decision making. Such an allocation is typically long term and leads to the underutilization of spectrum [1], [2].

Recent advances in wireless communications technologies have brought new approaches to the way radio devices might handle unwanted interference. The emergence of software defined radio concepts and proposed cognition capabilities for future radios seem to make possible distributed schemes for coexistence and cooperation of many closely located frequency agile communicating devices operating in same shared spectrum. The success of the recent regulatory "unlicensed band" experiments in the ISM bands have further brought into question the need for static allocation of spectrum portions for purposes of mitigating interference.

The above mentioned considerations have started an ongoing debate regarding the possible alternatives to the spectrum governance policy currently enforced by the US FCC [2]. The alternative governance schemes considered by the research community fall under two broad categories: (i) a market based approach with emphasis on the spectrum property rights model, and (ii) the spectrum commons model. Inspired by the landmark work of Coase [3], the spectrum property rights approach advocates, in the broadest sense, that spectrum can be treated just like land, and private ownership of spectrum is viable. The spectrum commons, on the other hand, proposes that spectrum should be treated like common property, and all communicating parties should have access to it as long as they abide by some usage rules.

The lack of conclusive arguments in this debate has led to recent calls [2], [4] for the development of practical spectrum access models to help in the debate. Nevertheless, a positive outcome of the debate has been the recognition to varying degrees, the advantages of employing dynamic access as well as market based spectrum allocation. This entails development of schemes that embody the dynamic nature of shared managed access to spectrum commons and also support the exclusivity of spectrum property rights.

In [5], we have proposed such an "Intermediate Model" in which service provider competitions were modeled in a shared managed access setting. The spectrum access model proposed made use of dynamic access schemes and employed market tools for spectrum allocation decisions while also promoting exclusive usage on a temporary basis.

In this paper we present another such intermediate scheme that retains a stronger bias toward the spectrum property rights approach while also retaining aspects of dynamic and competitive access. We call this scheme D-Pass (Dynamic 
Property Rights Spectrum Access). We focus more on the technology and network economics issues and do not go into issues that would otherwise be relevant to a property rights regime, such as the dispute resolution algorithms, secondary markets or flexibility of usage.

Coordinated spectrum access in a dynamic setting requires the presence of a central controller responsible for mediating between spectrum consuming parties. In [5]-[7], a spectrum policy server (SPS) was introduced to serve as such an enabler for coordinated spectrum access. The SPS is a central server responsible for coordinating spectrum access in a specified geographical region. It functions as a controller/enforcer as well as a clearinghouse for spectrum allocations. The SPS's role can also be extended to that of a spectrum broker [8], [9] who coordinates spectrum access across different serving areas or heterogenous devices in a geographical area.

The D-Pass model proposes a framework for dynamic allocation of spectrum resources and competitions among the operators (service providers) serving a specified serving area. Various other dynamic spectrum allocation schemes managing spectrum access across service providers have also been studied [10], [11]. [12] develops a spectrum allocation scheme based on spectrum auctioning among the operators, however it does not consider operator competitions for the users in the system.

The allocation of spectrum resources in the D-Pass model is based on session(s) based ownership of spectrum portions by the operators. The operators purchase portions of spectrum from the SPS and pay for the amount of bandwidth they secure whether or not they actually utilize all of it. The spectrum purchases are valid for a single communication session or possibly more. In the rest of the paper, unless specified otherwise, we consider spectrum allocation systems in which allocations are valid for one communications session only. No operator can use the portion of spectrum which is assigned to its competitors. In this sense the spectrum resource can be considered under the framework of property rights with short term dedication. The SPS optimally determines how much bandwidth each operator can own based on the maximization of a predefined objective function. The objective can be any system related metric like the expected bandwidth utilization in the system, metrics relating to mean user appreciation for the provided service, total revenue collected by the SPS or the like.

Given any specific bandwidth partition among the operators, the operators try to attract users through demand responsive pricing [13], [14]. Enjoying differing service spectral efficiencies $r[b p s / H z]$ for any given user, they make rate $R[b p s]$ offers in exchange of a price $P$ [units] for the given user. The user's willingness to accept the service is modeled through an acceptance probability $A(R, P)$. Each offer an operator makes to a given user invokes an expected income to the operator associated with the $A(R, P)$ as well as the price asked $P$, the related fixed operational costs (independent of the offered rate $R$ ). The operator's total profit depends on the total expected income it achieves from serving the users and its payment for the spectrum portion it was allocated by the SPS.

The operator competition for the users is modeled in the form of an iterative bidding scheme inspired by the simultaneous ascending auction [15], [16]. The operators make vectoral offers of $\vec{R}$ in exchange of vectoral prices $\vec{P}$ at each round, with each component of the $\vec{R}$ and $\vec{P}$ vectors denoting the rate offer and the price asked for the corresponding user. In each bidding round, the operators try to achieve the greatest acceptance probabilities for the users they would benefit from serving, while also maximizing their expected profits. In making their rate offers, the operators are obliged not to consume more bandwidth than that of the spectrum portion allocated by the SPS. The bidding process is terminated the first round there are no new bids for any user.

\section{D-PAss Model}

We consider a limited interference region with $N$ users who are served by $M$ operators. The available bandwidth of $W_{A}$ $\mathrm{Hz}$ is controlled by the SPS who partitions all or part of it among the competing operators in the form of non-overlapping portions. We assume each operator has a predefined number of base stations in the region of interest that are fixed in location. The user locations are not fixed, and keep changing at regular time intervals.

The allocation of the spectrum resources is valid for the duration of one communications session only. The sessions between the operators and the users are assumed to be synchronously initiated and finalized. In this work we assume each instantiation of user locations mark the start of a new communication session which lasts till a change in locations of one or more users is detected. Consequently, any change in user locations prompts a new spectrum allocation to take effect.

The potential customers/users in the system have no long term subscription to any operator. The operators compete for the users at the initiation of each communication session and the user-operator associations achieved at the end of the competition are valid for the immediate communication session only.

We consider an "interference free" system in which users are served in non-overlapping spectrum portions, i.e. at each point in the frequency spectrum, there is at most one useroperator pair communicating at a time.

The final spectrum allocation among the operators for any given session is the result of a hierarchical (two-tier) optimization process. In the upper tier, the SPS iteratively declares allocation vectors each of which induces an operator competition in the lower tier. The operator competition results in winning rate and price offers as well as user acceptance probabilities. The SPS iteratively determines the optimal allocation vector which maximizes the relevant objective function considered. Fig. 1 illustrates this iterative optimization process.

In the following, we describe in detail the user acceptance, operator income and spectrum allocation models that will govern the customer preferences, operator optimizations and bandwidth partition decisions for the D-Pass scheme proposed. 


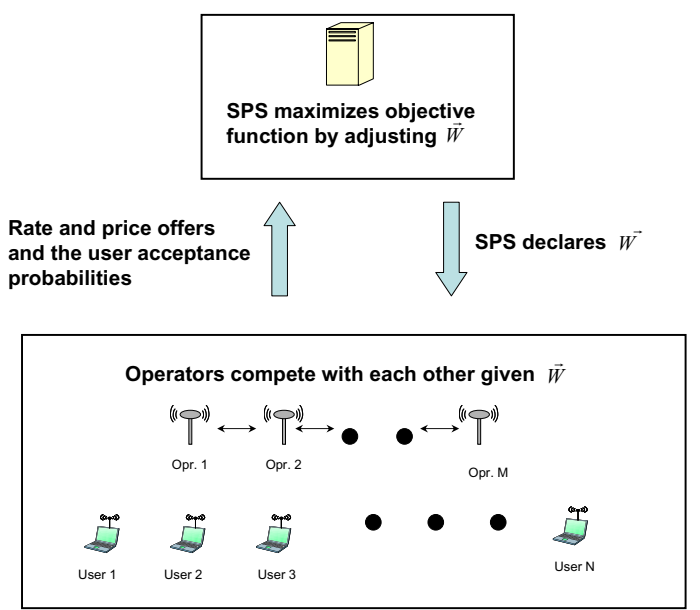

Fig. 1. SPS based hierarchical optimization for maximizing the specified system related objective function.

\section{A. Users' Acceptance Model}

In demand responsive pricing [13], [14], it is important to take into account the users' responses to the pricing strategy of the operator. From the user point of view, the service offer made by the operator is acceptable only if the price asked is reasonable. In [5], this consideration has been addressed through the use of the acceptance probability function which assigns acceptance probability values to a given rate-price offer pair for a given user. In this section, we present a basic description of acceptance probability that can also be found in [5].

Intuitively, the acceptance probability $A(R, P)$ should have the following qualitative properties. It should be an increasing function of the rate $R$ the user enjoys for a fixed price asked $P$ while decreasing in $P$ for fixed $R$. Mathematically, these properties are formulated as:

$$
\begin{array}{lcl}
\frac{\partial A}{\partial R} \geq 0, & \frac{\partial A}{\partial P} \leq 0, & \\
\forall P>0, & \lim _{R \rightarrow 0} A(R, P) & =0, \\
& \lim _{R \rightarrow \infty} A(R, P) & =1, \\
\forall R>0, & \lim _{P \rightarrow 0} A(R, P) & =1, \\
& \lim _{P \rightarrow \infty} A(R, P) & =0 .
\end{array}
$$

While there are several candidate choices for the function $A(R, P)$, we will follow [13], [14] and choose

$$
A(R, P)=1-e^{-C u(R)^{\mu} P^{-\epsilon}}
$$

where $\mu$ is the utility sensitivity of the user, $\epsilon$ is the price sensitivity, and $C$ is an appropriate constant. $R$ affects the acceptance probability through $u(R)$ which stands for the utility a user achieves given it communicates with rate $R$. In this work we consider a typical utility expression as expressed in (3).

$$
u(R)=\frac{(R / K)^{\zeta}}{1+(R / K)^{\zeta}}
$$

where $K$ and $\zeta$ are parameters that determine the exact shape of the above sigmoid function. Note that the above expression gives normalized utility values in the interval $[0,1)$ with the rate $R=K$ yielding a utility of $1 / 2$.

Note that the acceptance probability function can be differentiated among users through the above parameters. In this sense, the above acceptance model is similar to the CobbDouglas curves that are used in economics [17].

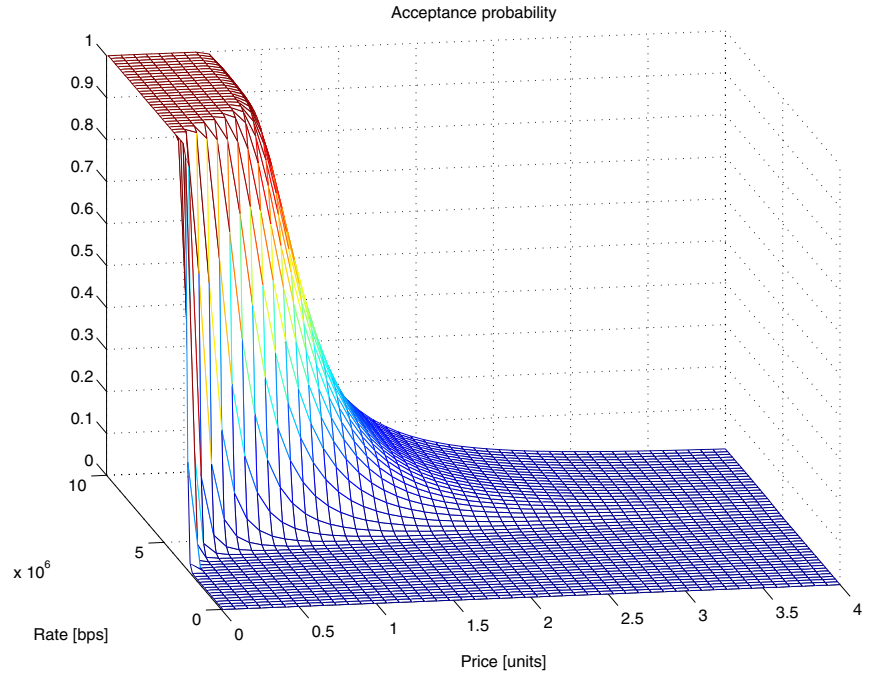

Fig. 2. The acceptance probability for $K=5 \times 10^{6}, \zeta=10, C=1$, $\epsilon=4, \mu=4$.

Fig. 2 illustrates the acceptance probability surface as a function of offered rate $R$ [bps] and asked price $P$ [units] for the parameter values $K=5 \times 10^{6}, \zeta=10, C=1, \epsilon=4$ and $\mu=4$.

\section{B. Operator Income Model}

It is beneficial for the operators to stay in operation only if the total expected profit they achieve serving the users compensates the payments to the SPS for the bandwidth portion purchased as well as the fixed operational costs involved in serving the users.

The individual expected income operator $i \in\{1, \ldots, M\}$ achieves serving any arbitrary user $n \in\{1, \ldots, N\}$ is expressed as:

$$
I_{i, n}\left(R_{i, n}, P_{i, n}\right)=A\left(R_{i, n}, P_{i, n}\right)\left(P_{i, n}-F_{i}\right),
$$

where $R_{i, n}$ and $P_{i, n}$ are the offered rate and price, respectively, corresponding to user $n$. $F_{i}$ [units] is the fixed operational cost incurred by operator $i$ while serving any user. Note that the fixed operational cost is incurred only if the service is provided, and it does not depend on the quality (amount) of service. In this sense, it is different from the sunk cost 
frequently encountered in pricing literature which refers to the type of cost that is incurred whether the service is provided or not.

A detailed discussion of the parameters involved in determination of $F_{i}$ for a given operator is beyond the scope of this work, but a relevant reference on cost estimation for further reading is [18].

Note that the total expected profit of an operator $i$ is the difference between the sum of the individual expected incomes from the users it serves and its payment for the bandwidth it has purchased from the SPS at the beginning of the communication session. This can mathematically be expressed as:

$$
\begin{gathered}
Q_{i}\left(\vec{R}_{i}, \vec{P}_{i}\right)=\sum_{n} A\left(R_{i, n}, P_{i, n}\right)\left(P_{i, n}-F_{i}\right)-W_{i} V, \\
i \in\{1, \ldots, M\}, \quad n \in \mathbf{N}^{\prime},
\end{gathered}
$$

where $n$ is the user index, and $\mathbf{N}^{\prime}$ is the set of users the specified operator makes offers to. $\left(\vec{R}_{i}, \vec{P}_{i}\right)$ are the offer vectors which specify the offers for each user. $W_{i}$ is the amount of bandwidth owned by the operator and $V$ [units/Hz] is the unit bandwidth cost the operators need to pay to the SPS for unit spectrum they purchase.

\section{Spectrum Allocation Model}

At the beginning of every communication session (with each change in user locations), the available spectrum is partitioned among the operators. Spectrum portions allocated to operators are non-overlapping, with $\sum_{i=1}^{M} W_{i} \leq W_{A}$, where $W_{A}$ is the total available bandwidth and $W_{i}$ is the bandwidth of the spectrum portion allocated to operator $i$. Note that, in such a "property rights" scheme where the operators need to make payments for the total amount of spectrum they buy, irrespective of the extent of utilization, it is possible for the operators to end up in a loss. Such a loss would be realized in case the total expected income the operator achieves as a result of the operator competition is not high enough to compensate for its payments to the SPS for the spectrum portions it purchases. This is much like a company making investments to enter consumer markets and facing bankruptcy due to wrong assumptions regarding the market conditions. In our work, we assume that SPS not only mediates the allocation of spectrum resources, but it also ensures a fair allocation in the sense that all operators are prevented from negative profits. Note that in order to accomplish this, the SPS might allocate zero bandwidth for those operators who would otherwise have negative profits, thus practically leaving them out of operation for the current session.

The exact bandwidth allocation vector $\vec{W}=\left[W_{1} \ldots W_{M}\right]^{T}$ is determined as a solution of the optimization problem where SPS maximizes one of the following: (1) total expected bandwidth utilization in the system; (2) the minimum acceptance probability that a user accepts the offered service. Note that there might be other choices for objective functions besides the ones we consider in this paper.
The SPS maximizes the objective function subject to the constraints that the total allocated bandwidth does not exceed the total available bandwidth $W_{\mathrm{A}}$ and that no operator ends up with negative profit for the current session. Consequently, the SPS optimization problem can be expressed as:

$$
\begin{aligned}
\max _{\vec{W}} & \operatorname{Obj}(., \vec{W}) \quad \text { st. } \sum_{i=1}^{M} W_{i} \leq W_{\mathrm{A}} \\
& Q_{i}^{S} \geq 0, \forall i \in\{1,2, \ldots, M\},
\end{aligned}
$$

where $\operatorname{Obj}(., \vec{W})$ is a generic notation for the objective function the SPS maximizes. $Q_{i}^{S}$ refers to the total expected profit of operator $i$ for the considered session. Note that the maximum achievable values for $\operatorname{Obj}(., \vec{W})$ depend on many parameters including the user locations, cost structures and the service spectral efficiencies of the operators.

The SPS optimization problem is solved using a sequential search method in which all combinations of bandwidth allocations among the operators are tested and the one which achieves the greatest objective value is chosen as the optimum allocation. For any tested allocation vector $\vec{W}$, the operators compete with each other for the users considering the bandwidth constraints imposed by the allocation vector, as illustrated for the case of $M=2$ operators in Fig. 3 .

The operators compete with each other in the form of an iterative bidding scheme that is reminiscent of simultaneous ascending auctions. In this scheme, details of which will be described later in the text, the operators make bids in rounds in the form of vectors $\left(\vec{R}_{i}, \vec{P}_{i}\right) \in R^{N+} \times R^{N+}$ where $i$ is the index for the operator making the offer. Each of the $N$ entries of the offer vectors $\left(\vec{R}_{i}, \vec{P}_{i}\right)$ specify the rate offers and the price asked by the operator $i$ for the corresponding user. The result of the operator competition determines at most one of the operators as the winner for each user, who then makes its respective winning service offer as the final offer.

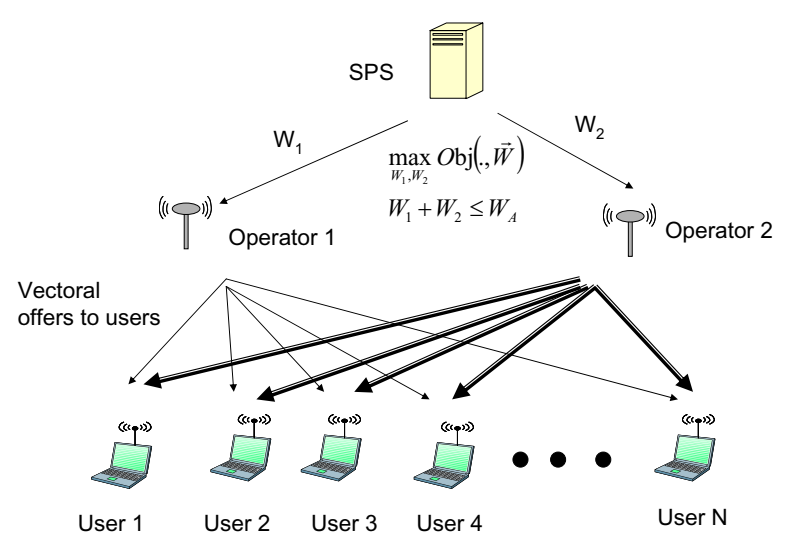

Fig. 3. SPS mediating iterative bidding processes among 2 operators for $N$ users. 


\section{SPS Objectives}

The formulation of the SPS optimization problem in (6) is generic enough to address many different objectives. In this work we have limited the problem to two objectives as mentioned earlier. We now present precise definitions for these objective functions.

1) Maximizing the Expected Bandwidth Utilization ( $E B U)$ : $\operatorname{EBU}(., \vec{W})$ is defined as the sum of the expected bandwidth utilizations of the users. In this sense, it is a function of the bandwidth allocation vector $\vec{W}$ as well as the user locations and the cost parameters in the system:

$$
E B U(., \vec{W})=\sum_{n=1}^{N} A_{n}^{f}(., \vec{W}) W_{n}^{f}(., \vec{W}) .
$$

In the above equation, $A_{n}^{f}$ and $W_{n}^{f}[H z]$ refer to the winning bid acceptance probability and bandwidth usage achieved for user $n$ as a result of the operator competition. $W_{n}^{f}$ depends on the winning rate offer $R_{\text {winner }}[b p s]$ and the winning operator's spectral efficiency $r_{\text {winner }, n}[b p s / H z]$ in serving the user, through the relation $W_{n}^{f}=R_{\text {winner }} / r_{\text {winner, } n}$.

2) Maximizing the Minimum Acceptance Probability $\left(A c p_{\min }\right)$ : The minimum acceptance probability is defined as:

$$
\operatorname{Acp}_{\min }(., \vec{W})=\min \left(A_{1}^{f}(., \vec{W}), \ldots, A_{N}^{f}(., \vec{W})\right)
$$

In maximizing the minimum acceptance probability, the SPS follows the max-min fairness criterion and achieves that acceptance probability vector $\overrightarrow{A^{f}}=\left[A_{1}^{f} A_{2}^{f} \ldots A_{N}^{f}\right]$ for which $A_{n}^{f}$ can not be increased without decreasing $A_{n^{*}}^{f}$ for some $n^{*}$ such that $A_{n^{*}}^{f} \leq A_{n}^{f}$. Thus in maximizing the above quantity the SPS emphasizes a fairer allocation as opposed to the EBU maximizing scheme.

\section{OPERATOR COMPETITION AND ITERATIVE BIDDING IN D-PASS MODEL}

We assume that in the presence of a number of service offers from different operators, any specified user accepts the service offer of the operator which induces the greatest acceptance probability for it, with the corresponding acceptance probability and ignores all other offers (practically setting their relevant acceptance probabilities to zero). Thus, in order to gain the right to serve any given user, an operator needs to make the offer which induces the greatest acceptance probability among all.

Consequently, the operator competition for the users can be modeled as an iterative bidding mechanism in which the operators make bids in rounds, in the form of rate and price offer vectors. The goal of the operators at each round is to come up with offers that would maximize their expected profits by inducing greater acceptance probabilities than those of the competing offers for each user.

Note that while competing for a number of users simultaneously, each operator is making use of the limited spectrum portion allocated to it, partitioning it among the rate offers it makes to different users. In this sense, the operator competition can be likened to a situation in which a number of goods (users) are to be partitioned among a number of buyers (operators) with budget constraints. The multi-item auction theory in economics literature [19] presents many different mechanisms through which multiple items can be assigned to numerous bidders in such settings.

In this work, we have developed a bidding mechanism which is similar to simultaneous ascending auctions [15], [16] in terms of implementation. Even though simultaneous ascending auction mechanisms do not always achieve the optimal operating points, as frequently mentioned in auction theory literature, we believe it is a good match for wireless communications settings due to the simplicity of the mechanism.

Below, we first present a brief discussion on simultaneous ascending bid auctions before proceeding to provide a detailed overview of the proposed bidding scheme considered here.

\section{A. The Simultaneous Ascending Auction}

The simultaneous ascending auction was first developed in 1994 for use in the US FCC's spectrum auctions. It is a simple extension of the single item ascending (English) auction to the case in which a number of bidders (buyers) simultaneously compete for multiple items.

The bidding occurs in rounds. At each round the bidders make price bids for the items they are interested in. At the end of each round the auctioneer declares the "standing high bid" and the corresponding highbidder, for each item. The auctioneer also declares the minimum bid for each item for the next round, as the sum of the standing high bid and a predetermined bid increment for the item. The predetermined bid increments are often the larger of a fixed amount and a fixed percentage (usually $5 \%$ or $10 \%$ ) of the standing high bid [15], [16]. A participant who is not the current highbidder for an item it is interested in, needs to increase it's price bid next round to exceed the current standing high bid by at least the bid increment amount, in order to be in the winning position. Note that the enforcement of the minimum bid rule aims at avoiding lengthy bidding periods in which bidders exceed the standing high bids only by negligible amounts each round. The bidding is finalized at the first round in which no bidder can raise its bid on any of the items anymore. Each item is awarded to the bidder who holds the current standing high bid for the item.

In such auction settings, the bidders can often end up with only part of what they desire. Such situations can often lead to the exposure problem in which the bidder ends up with items that are not useful to it by themselves only, and only are profitable to own in the company of some other items which have been sold to different parties. A simple example is an operator who wins only one of the two adjacent spectrum portions which is not sufficient for profitable operation by itself. In order to avoid this problem, in most versions of the simultaneous ascending auctions, the bidders are allowed to withdraw their bids. Such withdrawals, however are often 
punished with penalties. In some other versions, bid withdrawal is not allowed. The bid for any item is considered as a commitment by the bidder.

The simultaneous ascending auction mechanisms also specify rules regarding the activity and eligibility of the bidders. Interested readers can find discussions relevant to these rules in [15], [16].

\section{B. The Iterative Bidding}

Inspired by the generic rules cited above for a simultaneous ascending auction, we propose a simple bidding scheme, as a means of operator competition.

In the proposed bidding scheme, the operators make bids in rounds. The bids are in the form of rate and price offer vectors. At any given round, for any specified user $n$, the offer which induces the greatest acceptance probability is declared by the SPS as standing high acceptance probability $\left(S H A P_{n}\right)$ for that user. At the end of each round, the SPS computes the minimum acceptance probabilities $\left(M A P_{i, n}\right)$ for the next round for each user-operator pair considering the standing high acceptance probability $S H A P_{n}$ for the user and the minimum acceptance probability increments enforced $\left(M A P I_{i, n}\right)$ for any given operator $i$ regarding user $n$.

Note that $M A P I_{i, n}$ may differ for different operators for a given user $n$, as the operator who is currently the highest bidder ( $S H A P$ bearer) need not increase its acceptance probability in the next round, while the opponent operators need to exceed $S H A P_{n}$ by at least some predetermined value $\delta_{n}$ in order to claim the user. $\delta_{n}$ can be considered as an auction design parameter and is determined by the SPS. In case a number of operators simultaneously induce the $S H A P$ for the user in any given round, only one of them (randomly determined by the SPS) is treated as the current winner, while the rest are obliged to make more attractive offers for the user in the next round in order to be in the winning position. The SPS declares the $M A P$ values for the next round in the form of $M \vec{A} P_{i} \in[0,1)^{N}$ vectors where each element in $M \vec{A} P_{i}$ refers to the minimum acceptance probability operator $i$ needs to induce next round in order to be in the winning position for the corresponding user.

The iterative bidding is initialized by allowing the operators to choose their service offers without consideration of the opponent strategy. It is finalized the first round in which there is no new offers (no increase in $S H A P$ ) for any of the users.

A technical issue related to the $M A P I$ policy is the fact that for any positive price $P>0$, the acceptance probability, by definition, is always less than $1 ; A(R, P)<1$.

Considering the above, we define the $M A P$ for any user $n$ and operator $i$ as:

$$
M A P_{i, n}=\min \left(S H A P_{n}+M A P I_{i, n}, A_{\max }\right)
$$

where $A_{\max }<1$ is a predefined constant, which is set as close to 1 as possible. $S H A P_{n}$ is the standing high acceptance probability for the user from the previous round and $M A P I_{i, n} \geq 0$ is the minimum acceptance probability increment for operator $i$ regarding user $n$, declared by the SPS. $M A P I_{i, n}$ is defined as:

$$
M A P I_{i, n}= \begin{cases}0 & \begin{array}{l}
\text { if operator } i \text { has the } \\
\text { greater bid from last round, } \\
\text { otherwise, }
\end{array}\end{cases}
$$

where $\delta_{n}>0$ is the increment amount set by the SPS. There can be different approaches for setting $\delta_{n}$. In our numerical experimentation we have considered increasing increments, where $\delta_{n}$ is determined as a certain percentage of the $S H A P_{n}$, as explained later in the numerical results section.

We further impose the rule that if any operator happens to make an offer inducing acceptance probability $A_{\max }$ for any user, it wins the competition for the user and the other operators may no longer make any offers for the specified user. In case more than one operator makes the offer inducing acceptance probability $A_{\max }$ for the first time simultaneously, one of them is chosen by the SPS as the winner randomly. The SPS declares the user for which the auction is finalized this way in the form of a boolean vector $\vec{K} \in\{0,1\}^{N}$. For those users for whom the competition is finalized, the corresponding element of $\vec{K}$ is set to zero, while for others it is set to 1 .

While making their bids, the operators consider their costs and maximize their expected profit at each round, subject to the bandwidth constraints set by the allocation vector and the bidding rules mentioned above. As in some versions of simultaneous ascending bid auctions, we enforce the additional rule that the current $S H A P$ inducing offers should not be withdrawn; if operator $i$ is the one who has achieved the $S H A P_{n}$ for the specific user $n$ in the previous round, it can not make an offer which implies a lower acceptance probability than $S H A P_{n}$ for that user, otherwise it is penalized (with negative infinity payoff).

Considering the rules cited above, the total expected profit optimization problem for any operator at each round can be mathematically expressed as:

$\max _{\vec{R}_{i}, \vec{P}_{i}}\left(\sum_{n=1}^{N} \beta_{i, n}\left(R_{i, n}, P_{i, n}\right)-W_{i} V\right)$ st. $\sum_{n=1}^{N} W o_{i, n} \leq W_{i}$.

In the above formulation, $i$ is the index for the operator. $\vec{R}_{i}$ and $\vec{P}_{i}$ refer to the offered rate and price vectors for the operator, respectively. $W_{i}$ is the spectrum portion allocated to operator $i$ and $\beta_{i, n}$ is a function which reflects the expected income from user $n$ for operator $i$ as defined in (4), subject to the rules of the iterative bidding cited above. $W o_{i, n}$ is the bandwidth consumption relevant to the rate offer for user $n$.

$\beta_{i, n}$ can mathematically be expressed as follows: 


$$
\beta_{i, n}\left(R_{i, n}, P_{i, n}\right)= \begin{cases}0 & \text { if }\left(A\left(R_{i, n}, P_{i, n}\right)<M A P_{i, n}\right. \\ & \text { or } \left.\vec{K}_{n}=0\right) \text { and } M A P I_{i, n} \neq 0 \\ -\infty & \text { if } A\left(R_{i, n}, P_{i, n}\right)<M A P_{i, n} \\ & \text { and } M A P I_{i, n}=0 \\ A\left(R_{i, n}, P_{i, n}\right)\left(P_{i, n}-F_{i}\right) & \text { if } A\left(R_{i, n}, P_{i, n}\right) \geq M A P_{i, n},\end{cases}
$$

where $M A P_{i, n}$ stands for minimum bid required for user $n$ regarding operator $i$, as defined in (9). $W o_{i, n}$ can be expressed as:

$$
W o_{i, n}=R_{i, n} / r_{i, n}
$$

where $r_{i, n}[b p s / H z]$ is the spectral efficiency of operator $i$ for user $n$.

Note that the definition implies that for any given user $n$, if the operator does not exceed the $S H A P$ by the minimum increment even though it is not the $S H A P$ bearer from the previous iteration, or if the competition for the user is already blocked by the SPS, the operator will gain zero income. If the operator is the $S H A P$ bearer from the previous round and it lowers the acceptance probability it has induced in the previous iteration, it is penalized by receiving negative infinity payoff. An operator who is either the $S H A P$ bearer from the previous round (and does not lower the acceptance probability), or who successfully induces greater acceptance probability for the user, than that of the $S H A P$ from the previous round, simply achieves the expected income as defined in (4).

We now state the following theorems to develop some intuition regarding operator preferences while making their offers.

Theorem 3.1: For any operator $i$, the solution of the optimization problem (during the operator competition) in (11) satisfies the constraint with equality; $\sum_{n=1}^{N} W o_{i, n}=W_{i}$.

Proof: Consider any fixed price offer vector $\vec{P}_{i}$ and any rate offer vector $\vec{R}_{i}$ such that $\sum_{n=1}^{N} W o_{i, n}<W_{i}$. Assume that the rate offer for an arbitrary user $a$, is increased from $R_{i, a}$ to $R_{i, a}+\Delta$ by some positive $\Delta>0$. This results in a corresponding increase in the associated acceptance probability from $A\left(R_{i, a}, P_{i, a}\right)$ to $A\left(R_{i, a}+\Delta, P_{i, a}\right)$. Considering (12), it is clear that such an increase would potentially also increase $\sum_{n=1}^{N} \beta_{i, n}\left(R_{i, n}, P_{i, n}\right)$, not altering the second term $\left(W_{i} V\right)$ of the objective function in (11). Consequently the overall effect of such an increase would be an increase in the achieved total expected profit. Thus, the operator would keep increasing offered rates as long as the allocated bandwidth is not exceeded. In the special case when the considered operator may not further increase the induced acceptance probability for any user (the acceptance probabilities have already reached $\left.A_{\max }\right)$, then the operator can increase both the offered rate and price so that the induced acceptance probability does not change. Inspection of (12) reveals that these increases would still increase the expected profit for the operator. Thus, in this special case the operator would again keep increasing offered rates as long as the allocated bandwidth is not exceeded. It is thus shown that, during the operator competition, the solution of the operator optimization problem should always saturate the constraint $\sum_{n=1}^{N} W o_{i, n} \leq W_{i}$.

The above theorem shows that in all rounds, both operators will offer all of the spectrum portions allocated to them by the SPS. This can be considered as a natural result of operating in a property rights like regime; since the operators are charged for all of the spectrum portions they control, irrespective of the extent of utilization, it is always better to fully utilize them.

Theorem 3.2: For any given fixed system geometry (user locations and number of users ) and fixed operational costs $F_{i}, i \in 1,2, \ldots, M$, the maximum achieved value for any one of the two considered objective functions is non-increasing in unit bandwidth cost $V$ [units $/ H z] ; O b j^{*}(V,.) \geq O b j^{*}(V+\Delta,$. where $\operatorname{Obj}^{*}(V,$.$) is the maximum achievable value for the$ objective function and $\Delta>0$ is a constant.

Proof: Consider the operator optimizations in (11). Note that the solution of the operator optimization problem does not depend on $V$, as $V$ is only included in the second term of the objective function which does not involve any of the optimization parameters. Consequently, for any declared $\vec{W}$, the value of objective function $\operatorname{Obj}(., \vec{W})$ does not depend on $V$. On the other hand, the resulting operator profits diminish with increasing $V$ as their income from the users do not change and payments to SPS increase linearly with $V$. Thus, the space of allocation vectors $\vec{W}$ for which the operators achieve non negative total profits diminish with increasing $V$. Consequently, the optimization domain for the SPS in optimization problem (6) shrinks, either decreasing the maximum achieved value for the objective function; $\mathrm{Obj}^{*}(V,)>.O b j^{*}(V+\Delta,$.$) or$ at best keeping it unchanged; $O b j^{*}(V,)=.O b j^{*}(V+\Delta,$.$) .$

This theorem shows that increasing unit bandwidth cost potentially hurts the SPS objectives considered in this paper.

\section{LONG TERM AllocAtion OF SPECTRUM RESOURCES IN D-PASS MODEL}

So far the scheme we have described considers spectrum resource allocation for a single communication session only. We now briefly describe how this scheme could be extended to address those cases in which the spectrum allocation is considered for longer durations. Recall that the communication session is defined to be the duration between consecutive changes in user geometry. In this sense, a longer term allocation of resources would be valid for a number of consecutive changes in user geometry.

We parameterize the duration of spectrum allocation decision by $T$ which denotes the number of communication sessions for which the decisions are valid. Note that with increasing $T$, the scheme approaches to a static allocation approach.

The bandwidth allocation vector chosen by the SPS is valid for $T$ sessions in a row. Although the SPS decisions are made considering $T$ sessions, we assume that the operators still compete for users at each instantiation of user locations (only the spectrum management is in the form of static governance 
for the $T$ sessions), through the iterative bidding described in section III-B . Consequently, the long term SPS optimization problem can be expressed as:

$$
\begin{aligned}
\max _{W_{T}} \overline{O b j_{T}}\left(., \vec{W}_{T}\right) \quad \text { st. } \sum_{i=1}^{M} W_{i} \leq W_{\mathrm{A}} \\
Q_{i}^{T} \geq 0, \forall i \in\{1,2, \ldots, M\}
\end{aligned}
$$

where $\vec{W}_{T}$ refers to the allocation vector which is valid for $T$ communication sessions and $Q_{i}^{T}$ is the final profit of operator $i$ at the end of $T$ sessions, which is defined as the sum of the session based profits for each of the $T$ sessions. $\overline{O b j_{T}}$ is the average value for the SPS objective function considered over $T$ sessions.

Note that the above described operation model is similar to the operation of present day service providers. The service providers purchase licenses for spectrum portions from the FCC for long durations (approximately 10 years, subject to extension). Their decision to actually purchase the spectrum portions at the requested prices depends on their long term profitability. However, they keep changing their market penetration strategies several times during the 10 years they have the right to use the specified spectrum portions.

\section{NUMERICAL EXPERIMENTS}

In this section we provide numerical results corresponding to the D-Pass schemes described earlier in the text. In our experimental setup, we consider $M=2$ operators located in a simple linear region. Each operator has only one base station. Each change in user locations denotes the beginning of a new communications session. The linear region and the locations of the base stations are as depicted in Fig. 4, which shows an instantiation of user locations for 8 users.

The spectral efficiency between base station $i$ and the mobile terminal $n$ is determined as

$$
r_{i, n}=\log _{2}\left[1+\frac{P_{\mathrm{s}}}{N_{o}}\left(\frac{d_{i, n}}{L / 4}\right)^{-2}\right],
$$

where $P_{\mathrm{s}}$ is the signal power, $N_{o}$ is the AWGN variance, $d_{i, n}$ is the distance between the base station (operator) $i$ and the terminal, and $L$ is the total length of the linear region in Fig. $4(L=1000 \mathrm{~m})$. We set $P_{\mathrm{s}}=2 N_{o}$, which guarantees a $\mathrm{SNR}=3 \mathrm{~dB}$ at the distance of $L / 4=250 \mathrm{~m}$ from the base station.

The available bandwidth considered is $W_{A}=10 \mathrm{MHz}$ and the users are assumed to have utility parameters used in Fig. 2.

In order to keep the exhaustive search in SPS optimization tractable, the bandwidth is quantized to be made of basic units of approximately $380 \mathrm{kHz}$ wide.

Recall that the fixed operational cost $F_{i}$ for operators 1 and 2 can be a complicated function of many parameters including the number of base stations, physical layer technology used and the like. In this paper, for the sake of simplicity we consider a symmetric cost structure with $F_{1}=F_{2}=F$

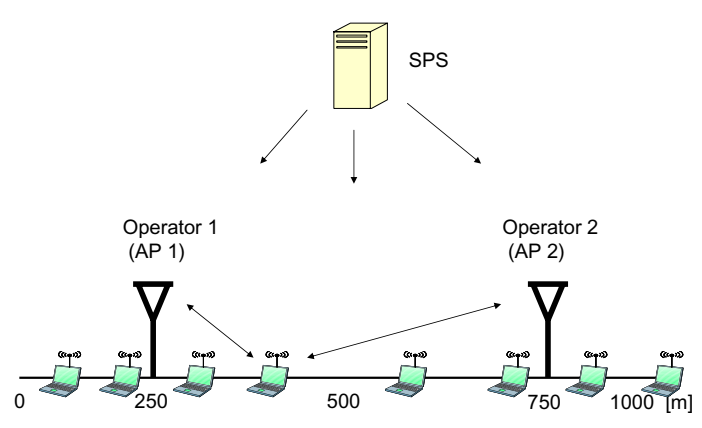

Fig. 4. Geographical region with two operators.

[units]. We also assume that the SPS will be charging both operators at the same variable cost rate $V[$ units $/ \mathrm{Hz}]$.

During our experimentations, we have considered different increment $(M A P I)$ policies; including increasing increments with increment $\delta_{n}=\eta \times S H A P_{n}$, where $\eta$ is a predefined percentage. Note that in this approach the SPS imposed increments increase throughout the bidding period, since $S H A P_{n}$ increases (or stays the same) with each iteration. We have also considered diminishing increments where $\delta_{n}=\eta \times(1-$ $\left.S H A P_{n}\right)$. Note that in this approach, the increment is actually diminishing in each round. Our observation is that the specific choice of increment policy among these alternatives does not significantly affect the comparisons presented below. The plots included in this paper are achieved using the increasing increments with $\eta=10 \%$.

In the numerical experiments, we test three different schemes. As mentioned earlier in section II-D, we consider the expected bandwidth utilization (EBU) maximizing scheme and the minimum acceptance probability (Min. Acp.) maximizing scheme. For comparison purposes, we also consider the equal partition (EP) scheme in which each operator is allocated exactly half of the available spectrum, subject to the constraint that the operator achieves positive profit at the end of the competition, otherwise it is allocated no bandwidth.

We present plots for different cost ( $F$ and $V$ ) values for a 8 user system. Recall that the base station locations are fixed and the user locations are randomly determined assuming a uniform distribution for each user. Each data point is generated by testing 300 different instantiations of user locations (communication sessions). The results are then averaged over all 300 different realizations and the average values are presented in Figs. 5-10.

Fig. 5 shows the achieved expected bandwidth utilization in a 8 user system, as function of cost parameters $F$ and $V$, for the EBU maximizing scheme. It is observed that, the achieved expected bandwidth utilization is decreasing in both cost parameters $F$ and $V$. This observation is in accordance with Theorem 3.2. We also observe (not shown here) that the 


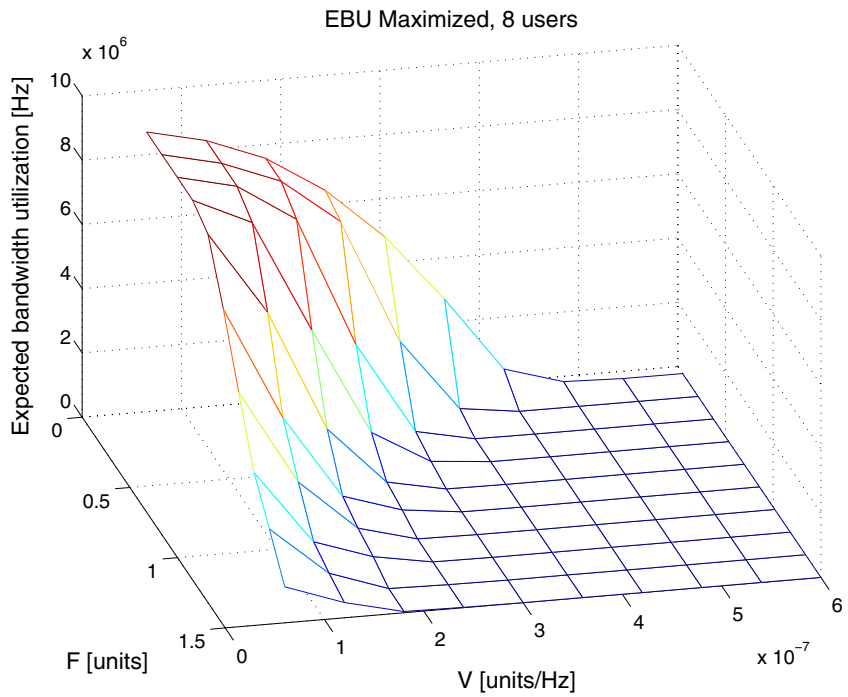

Fig. 5. Expected bandwidth utilization in EBU maximizing system in a 8-user system.

same decreasing pattern remains for the schemes where the SPS either maximizes the minimum acceptance probability or equally partitions the bandwidth.

To develop a better understanding for possible comparisons among the considered schemes as well as the effect of increasing cost on various performance metrics, we consider two different trajectories in the $F-V$ plane shown in Fig. 5. We conduct experiments for many $(F, V)$ pairs on these two trajectories that are shown in Fig. 6. One of the trajectories follows $(F, V)$ pairs along a line such that $V W_{A} / F=0.5$. The other trajectory follows $(F, V)$ pairs along a line such that $V W_{A} / F=4$. Note that the former trajectory reflects a cost structure in which the variable spectrum cost $V W_{A}$ has relatively lower weight against $F$, as opposed to the latter trajectory. Consequently, we refer to the first trajectory as the $F$-dominated trajectory and the latter one as the V-dominated trajectory throughout the rest of the paper.

We parameterize points on the trajectories by the total cost metric $F+V W_{A}$. Each value of $F+V W_{A}$ denotes a unique $(F, V)$ pair on the considered trajectory and increasing $F+$ $V W_{A}$ corresponds to progressing along the trajectory further away from the origin.

In Figs. 7 and 8, we present the expected bandwidth utilization and the average number of users served, as functions of the cost metric $F+V W_{A}$ [units], with $V W_{A} / F=0.5$ ( $F$ dominated) and $V W_{A} / F=4$ ( $V$-dominated), respectively. The average number of users served in the system refers to the number of users for which the final offered rates as well as the final acceptance probabilities are positive.

Figs. 7(a) and 8(a) show that with increasing cost metric the bandwidth utilization diminishes. The three schemes considered achieve very similar bandwidth utilizations for low values of the cost metric along both the $F$-dominated and $V$-dominated trajectories. As the cost metric is increased, it is observed that the schemes perform differently, with

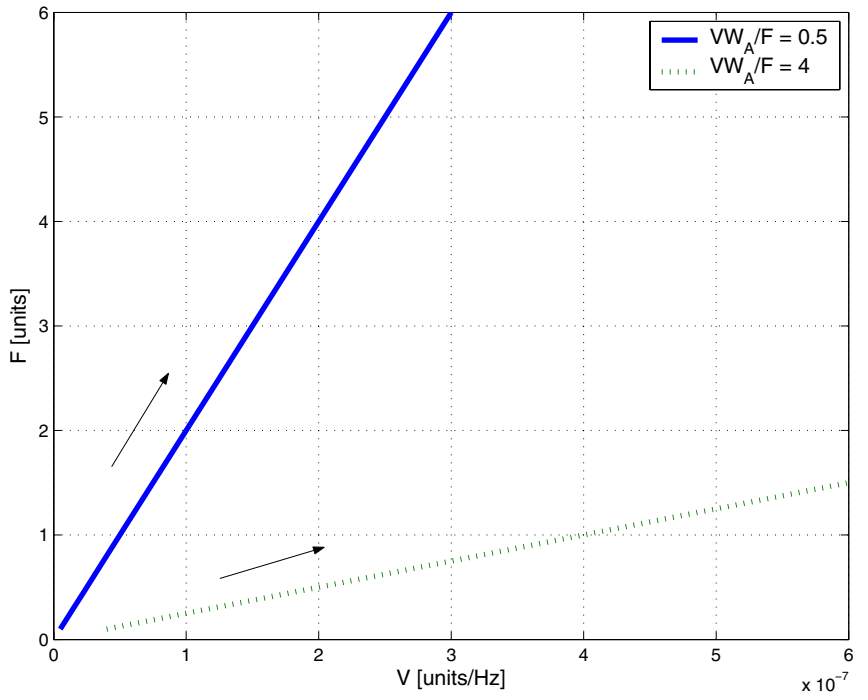

Fig. 6. Illustration of the trajectories.

the EBU maximizing scheme achieving the best utilization. The difference in the bandwidth utilizations of the schemes observed with increasing cost metric is more dramatic along the $V$-dominated trajectory. These results collectively suggest that the achieved bandwidth utilization becomes more sensitive to the specific scheme employed with increasing cost. It is seen that intelligent optimization schemes are more helpful when the unit bandwidth is relatively costly.

Figs. 7(b) and 8(b) show that the Min. Acp. maximizing scheme always achieves the greatest average number of users served in the system. This is intuitive since the Min. Acp. maximizing scheme promotes a max-min fairness criterion for the users. The number of users served is decreasing in cost metric for the Min. Acp. maximization and EP schemes, along both trajectories. It is observed that for low values of the cost metric $F+V W_{A}$, the EBU maximization scheme results in the lowest number of users served. For the EBU maximization scheme, as the cost metric is increased, a slight decrease is followed by an increase and a final decrease, along both trajectories. This pattern is more apparent along the $V$ dominated trajectory (see Fig. 8(b)), where it is observed that there is a cross-over between the curves for the EBU maximizing and EP schemes.

We now present an interpretation of the above trend regarding the average number of users served for the EBU maximizing scheme. Our detailed observations suggest that in the EBU maximizing scheme, the SPS is in the tendency of allocating most of the bandwidth to the operator who can serve users which enjoy greater service spectral efficiencies. This is much like a water-filling solution encountered in classical resource allocation, in which users with good channels are allocated more resources. Note that, considering the definition of EBU in section II-D, such an allocation would increase the EBU. Note also that, in the other schemes, there is no such incentive. Consequently, for low cost values, the SPS 


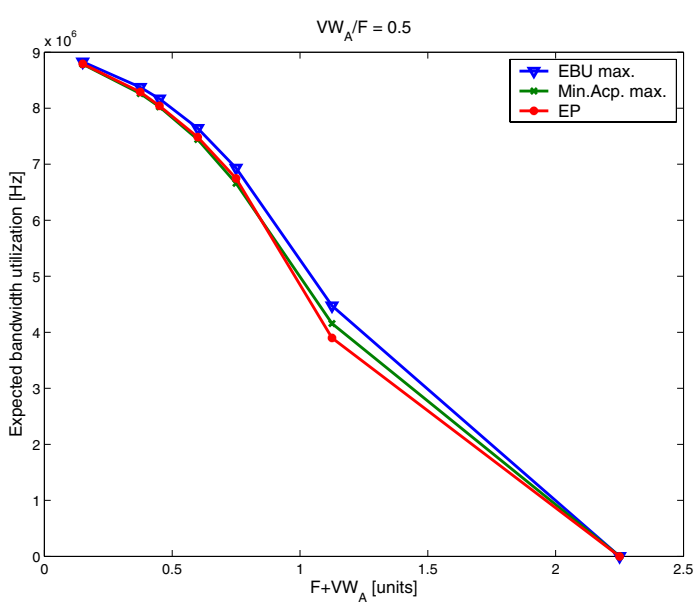

(a) Expected bandwidth utilization

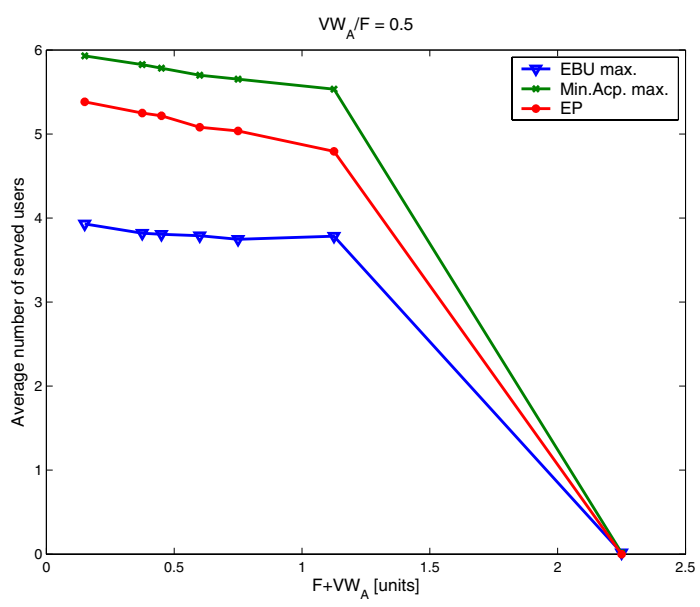

(b) Average number of users served

Fig. 7. F-dominated trajectory

partitions the spectrum among the operators in such a way that those users who are relatively further away from the access points are not served at all. This often means that the operator who is more likely to serve the distant users is allocated little bandwidth by the SPS, forcing it to effectively deny service to distant users and serve relatively low number of users. However, as the cost is increased, the operator that is allocated small spectrum portions is not able to maintain positive profit anymore. Thus the SPS is obliged to allocate more resources to such an operator, who in turn serves more users. This pattern is more evident in curves along the $V$-dominated trajectory simply because the variable cost $V W_{i}$ (for operator $i$ ) in (11) is the major factor in determining the affordability of spectrum allocations, as $F$ is compensated on a per user basis. As the costs are further increased, a decrease is observed since with even higher costs, the operators face diminishing returns and can not make convincing offers to the users.

Figs. 9(a) and 9(b) support the above interpretation. They show the total allocated bandwidth to the operators and the average difference between bandwidths of spectrum portions allocated to operators along the $V$-dominated trajectory for

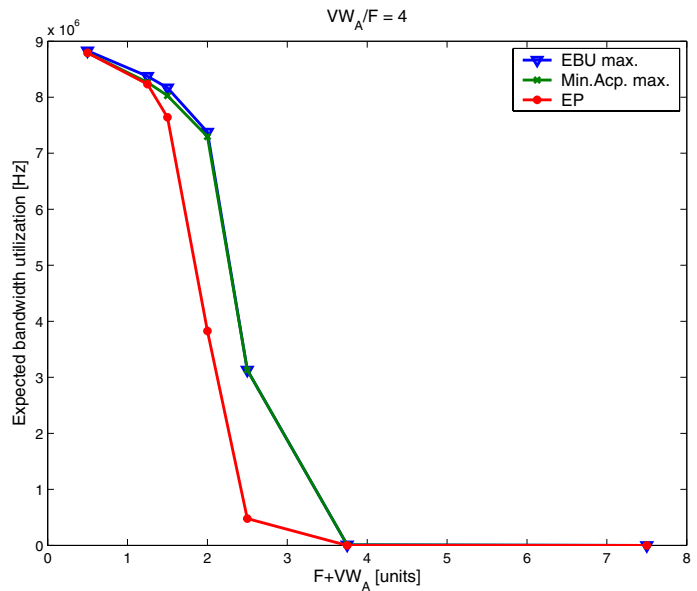

(a) Expected bandwidth utilization

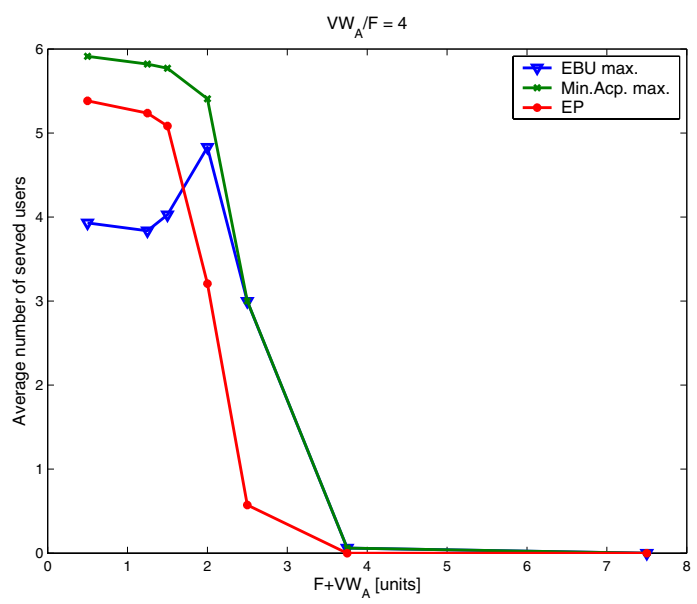

(b) Average number of users served

Fig. 8. V-dominated trajectory

the EBU maximizing scheme. The points on the curves corresponding to data points in Fig. 8(b) are labeled. It is easy to see that for the data points with low cost metric (points A,B,C,D), the average difference between spectrum portions for operators is considerably large, verifying the above intuition that the operator likely to serve distant users is given considerably less bandwidth. With increasing cost the total allocated bandwidth stays more or less constant for the first four data points (A,B,C,D), while the average difference is reduced, supporting the above intuition that spectrum resources are more fairly distributed in this region. This explains the increase in the average number of users served from $\mathrm{C}$ to $\mathrm{D}$ (see Fig. 8(b)). As the cost is further increased, the total allocated bandwidth diminishes, suggesting that in this region the high cost makes it difficult for operators afford spectrum portions allocated to them.

In Figs. 10(a) and 10(b), we present the illustrative results for longer term dedication of system resources in an EBU maximizing scheme for the $F$-dominated and $V$-dominated trajectories, respectively. The $T=1$ curves refer to the scheme in which the SPS updates the spectrum allocation 


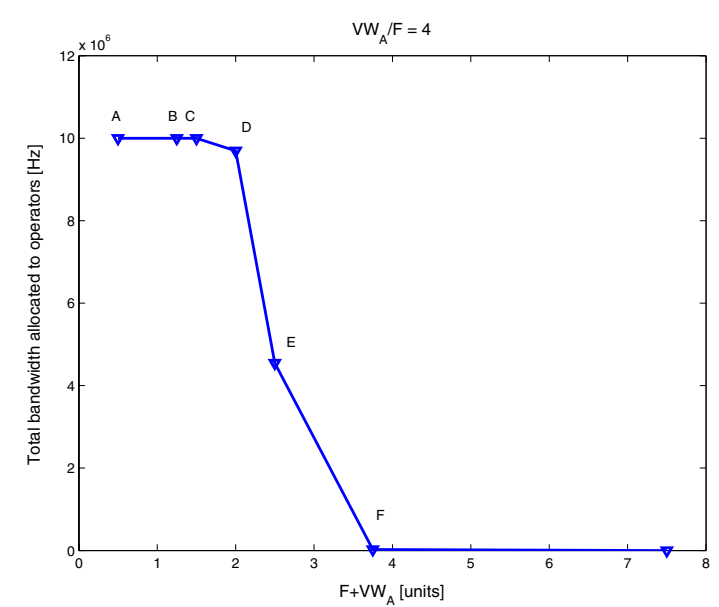

(a) Total bandwidth allocated

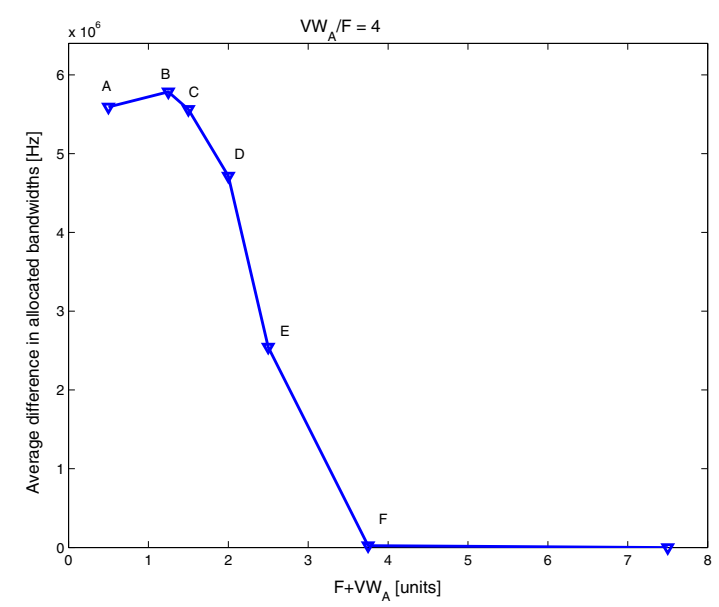

(b) Average difference in allocated bandwidths.

Fig. 9. Bandwidth allocation among the operators along V-dominated trajectory for the EBU maximizing scheme.

vector every communication session, e.g. every time a change in user locations is detected. The $T=5$ and $T=10$ curves refer to longer term spectrum allocation schemes in which the SPS updates the allocation vector every 5 sessions and every 10 sessions, respectively. Recall that no matter what $T$ is set to be, the operators compete with each other each time there is a change in user locations. The values plotted for $T>1$ refer to the average values per communication session.

As the updates in spectrum allocation become less frequent, the SPS is restricted to use the same allocation vector for greater number of sessions. This seems to constrain the SPS thus potentially reducing the achieved expected bandwidth utilization. On the other hand, the constraints on the final profits of the operators are relaxed as opposed to $T=1$, as the operators need to end up with positive profit only at the end of $T$ sessions, as opposed to every single session as in $T=1$. This seems to be in favor of increasing performance.

The plots shows that for the $F$-dominated trajectory, all three updating schemes achieve similar performance, with $T=$ 1 performing slightly better than the other two. This suggests

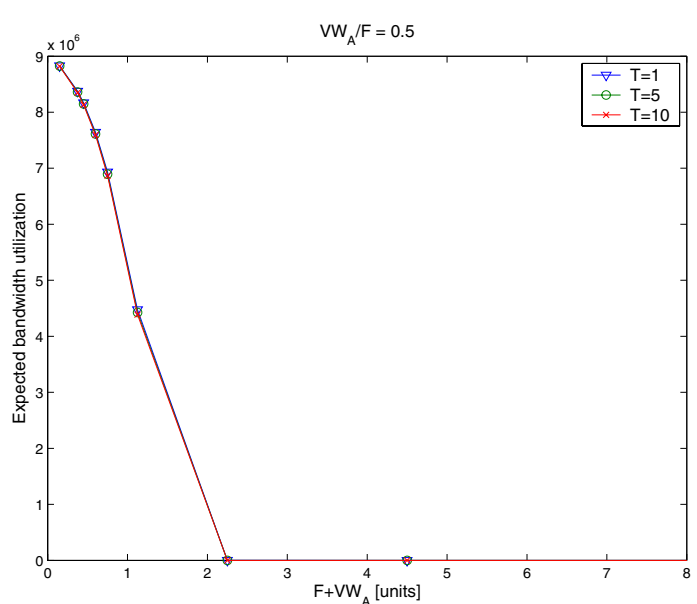

(a) $V W_{A} / F=0.5$

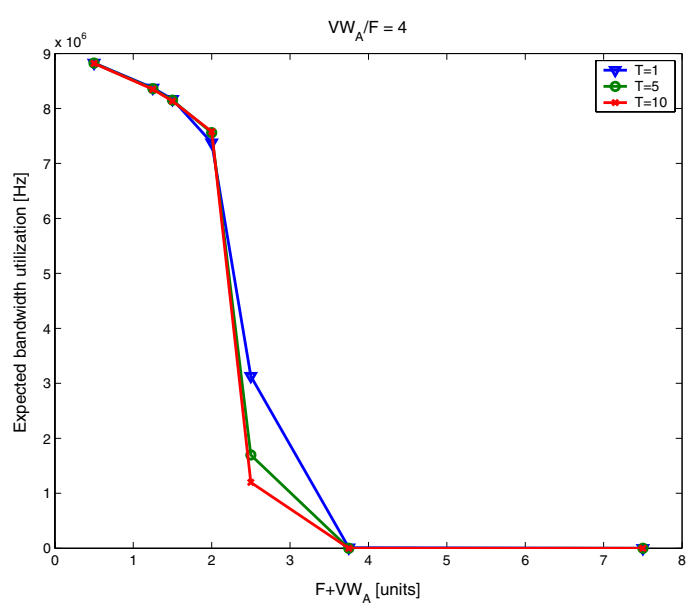

(b) $V W_{A} / F=4$

Fig. 10. Effect of long term dedication of spectrum resources for the EBU maximizing scheme.

that when the bandwidth is relatively inexpensive, the D-Pass scheme could be implemented over time durations comprised of several sessions, with negligible decrease in performance.

For the $V$-dominated trajectory, the $T=1$ scheme achieves significantly greater bandwidth utilization for high cost values. Thus, when the bandwidth is expensive, a clear tradeoff emerges between system performance (spectral efficiency) versus the duration of the short term allocation.

These plots, thus, suggest that employing short term allocation of resources could potentially lead to performance gains as opposed to more slowly changing (or static) allocation of resources, especially in regimes where the bandwidth is expensive.

\section{CONCLUSIONS}

In this paper we developed a framework for operator competition based on short term ownership of spectrum resources and devised a dynamic spectrum access method called "Dynamic Property Rights Spectrum Access (D-Pass)". The DPass model provides both the dynamic nature of managed 
shared access to spectrum commons, as well as the exclusivity of the spectrum property rights. It also serves as an example for the kind of practical spectrum access models that might help solidify discussions relating to the spectrum debate. In the D-Pass model, spectrum portions are allocated to operators by a SPS (Spectrum Policy Server) on a short term basis (a few sessions). Prior to each communication session, the SPS optimally determines a specific partition of spectrum resources among the operators to maximize a system related objective function. The operators are charged by the SPS for the amount of spectrum they are allocated. Given the spectrum allocation, the operators compete with each other for users present in the system through demand responsive pricing, in the form of an iterative bidding scheme reminiscent of simultaneous ascending auctions. At every iteration, the operators make rate and price offers for each user considering the bandwidth allocated to them and their costs associated with serving the users. The users respond by declaring the probabilities with which they will accept the service offers made. We considered two different objective functions for the SPS to maximize: (1) the total expected bandwidth utilization, (2) the minimum acceptance probability that a user accepts the offered service. We demonstrated several tradeoffs between these objectives through numerical experiments and illustrated the effect of bandwidth cost on these tradeoffs. Our results also suggest that employing short term allocation of resources could potentially lead to performance gains as opposed to static allocation of resources, especially in regimes where the bandwidth is relatively expensive.

\section{ACKNOWLEDGEMENT}

This work is supported in part by the NSF under grant number NeTS-0434854 and by the Defense Spectrum Office (DSO) of the Defense Information Systems Agency.

\section{REFERENCES}

[1] M. McHenry and D. McCloskey, "New York City spectrum occupancy measurements september 2004," Tech. Rep., December 2004. [Online]. Available: http://www.sharedspectrum.com/inc/content/ measurements/nsf/NYC_report.pdf

[2] J. M.Peha, "Approaches to spectrum sharing," IEEE Communications Magazine, vol. 43, no. 2, pp. 10-12, February 2005.

[3] R. H. Coase, "The federal communications commission," Journal of Law and Economics, vol. 2, pp. 1-40, Oct. 1959.

[4] G. R. Faulhaber, "The question of spectrum: Technology, management and regime change," in The Conference on the Economics, Technology and Policy of Unlicensed Spectrum, East Lansing, MI, 2005. [Online]. Available: http://quello.msu.edu/conferences/spectrum/program.htm

[5] O. Ileri, D. Samardzija, T. Sizer, and N. B. Mandayam, "Demand responsive pricing and competitive spectrum allocation via a spectrum server," in Proceedings of the IEEE International Symposium on New Frontiers in Dynamic Spectrum Access Networks (DySpan), Baltimore, MD, USA, 8-11 Nov., 2005, pp. 194-202.

[6] N. Mandayam, "Cognitive algorithms and architectures for open access to spectrum," in The Conference on the Economics, Technology and Policy of Unlicensed Spectrum, East Lansing, MI, 2005. [Online]. Available: http://quello.msu.edu/conferences/spectrum/program.htm

[7] C. Raman, R. Yates, and N. B. Mandayam, "Scheduling variable rate links via a spectrum server," in Proceedings of the IEEE International Symposium on New Frontiers in Dynamic Spectrum Access Networks (DySpan), Baltimore, MD, USA, 8-11 Nov., 2005, pp. 110-118.
[8] M. M. Buddhikot, P. Kolodzy, S. Miller, K. Ryan, and J. Evans, "Dimsumnet: New directions in wireless networking using coordinated dynamic spectrum access," in Proceedings of the IEEE WoWMoM05, 13-16 June 2005, pp. 78-85.

[9] M. Buddhikot and K. Ryan, "Spectrum management in coordinated dynamic spectrum access based cellular networks," in Proceedings of the IEEE International Symposium on New Frontiers in Dynamic Spectrum Access Networks (DySpan), Baltimore, MD, USA, 8-11 Nov., 2005, pp. 299-307.

[10] D. Grandblaise, D. Bourse, K. Moessner, and P. Leaves, "Dynamic spectrum allocation (dsa) and reconfigurability," in SDR Forum, San Diego, CA- Nov 2002.

[11] K. Moessner, J. Luo, E. Mohyeldin, D. Grandblaise, C. Kloeck, I. Martoyo, O. Sallent, P. Demestichas, G. Dimitrakopoulos, K. Tsagkaris, and N. Olaziregi, "Functional architecture of end-to-end reconfigurable systems," in Proceedings of the 63rd IEEE Vehicular Technology Conference (VTC), 2006, pp. 196-200.

[12] V. Rodriguez, K. Moessner, and R. Tafazolli, "Auction driven dynamic spectrum allocation: Optimal bidding, pricing and service priorities for multi-rate, multi-class cdma," in Proceedings of the IEEE International Symposium on Personal Indoor and Mobile Radio Communications (PIMRC), Berlin, Germany, 4-8 June, 2005.

[13] M. Lindstrom, "Demand responsive resource management for cellular networks," Ph.D. dissertation, KTH Royal Institute of Technology, Stockholm, Sweden, 2005

[14] L. Badia, M. Lindstrom, J. Zander, and M. Zorzi, "Demand and pricing effects on the radio resource allocation of multimedia communication systems," in Proceedings of the Global Telecommunications Conference (GLOBECOM), San Francisco, CA, USA, Dec., 2003, 2003.

[15] P. Milgrom, "Putting auction theory to work: The simultaneous ascending auction," 1997. [Online]. Available: citeseer.ist.psu.edu/milgrom99putting.html

[16] P. Cramton, "Simultaneous ascending auction," 2004.

[17] H. R. Varian, Intermediate Microeconomics: A Modern Approach. New York, W.W. Norton, 1987.

[18] C. Courcoubetis and R. Weber, Pricing Communication Networks. Wiley, John Sons, Incorporated, 2003.

[19] V. Krishna, Auction Theory. Academic Press, 2002. 